\title{
Avaliação da Secreção e Resistência Insulínica em Indivíduos com Diferentes Graus de Tolerância à Glicose - do Metabolismo Normal ao Diabetes Mellitus
}

artigo original

\author{
FERNANDA H. CORRÊA \\ VERÔNICA G. NOGUEIRA \\ MARIA de FÁtIMA BEVILÁCQUA \\ MARÍLIA DE BRITO GOMES
}

Disciplina de Diabetes e Metabologia, Departamento de Medicina Interna, Hospital Universitário Pedro Ernesto, Universidade do Estado do Rio de Janeiro - UERJ, RJ.

\section{RESUMO}

Objetivo: Os principais objetivos são determinar a associação entre os parâmetros clínicos e demográficos e os diferentes índices de secreção e resistência insulínica em indivíduos aparentemente saudáveis, sem conhecimento prévio de seu grau de tolerância à glicose. Pacientes e Métodos: Submetemos ao teste oral de tolerância à glicose (TOTG), no período de fevereiro a agosto de 2003, 105 indivíduos com média de idade de $33,4 \pm 1,4$ anos, sendo $57,1 \%$ do sexo feminino, subdividindo-os em 4 grupos: grupo 0 (normais): indivíduos com IMC $<25$ e metabolismo glicídico normal, grupo 1 (obesos): IMC $\geq 25$ e metabolismo glicídico normal, grupo 2 (IFG): glicemia de jejum alterada e grupo 3 (IOG): intolerância oral à glicose. Resultados: Encontramos diferença estatística para todas as variáveis analisadas durante o TOTG dentre os 4 grupos de indivíduos: glicemias de jejum e em 2 horas ( $p<0,05$; $p<0,05)$, valor de pico $(p<0,05)$, delta $(p=0,02)$, percentual de incremento $(p=$ $0,047)$, área sob a curva $(p \leq 0,05)$ e tempo de pico da glicose $(p=0,022)$. Não encontramos diferença para a velocidade de incremento da glicose, assim como para nenhuma variável da curva de insulina. Em relação aos índices de secreção insulínica, não houve significância estatística para os índices insulinogênico ou delta, porém estes tornaram-se significantes após correção da secreção pela resistência insulínica $(p=0,008)$. Quanto aos índices de resistência insulínica, os índices HOMA e QUICKI foram estatisticamente significativos $(p=0,005 ; p=0,005$, respectivamente), assim como a relação glicose/insulina em jejum $(p=0,053)$. Conclusão: Apesar do tamanho limitado da amostra, podemos inferir que indivíduos com intolerância à glicose em jejum e pós-prandial possivelmente estão em momentos diferentes da história natural da doença. Nossos dados demonstram que os melhores índices para a avaliação de resistência insulínica são o HOMA e o QUICKI, e que os índices de avaliação da secreção pancreática devem ser corrigidos para o grau de resistência insulínica, de modo a refletir melhor a história natural do diabetes mellitus. (Arq Bras Endocrinol Metab 2007;51/9:1498-1505)

Descritores: Resistência insulínica; Secreção insulínica; TOTG; Intolerância à glicose

\section{ABSTRACT}

Insulin Resistance and Secretion Assessment across a Range of Glucose Tolerance from Normal Individuals through Diabetes.

Aim and Methods: Our main aim was to determine the association between clinical, demographical parameters and different insulin resistance and secretion indices in apparently healthy subjects, without previous knowledge of their own level of glucose tolerance. For that purpose, we evaluated 105 individuals from February to August 2003 by means of OGTT, aged $33.4 \pm 1.4$ years old, $57.1 \%$ female. We allocated them in four groups: group 0 (normal): individuals with $\mathrm{BMI}<25$ $\mathrm{Kg} / \mathrm{m}^{2}$ and normal glucose metabolism, group 1 (obese): $\mathrm{BMI} \geq 25 \mathrm{Kg} / \mathrm{m}^{2}$ and normal glucose metabolism, group 2 (IFG): impaired fasting glucose and group 3 (IGT): impaired glucose tolerance. Results: We have found statistical difference on all variables during OGTT between all groups: fasting glucose $(p<0.05)$, 2-hour glucose $(p<0.05)$, glucose peak value $(p<0.05)$, glucose delta $(p=0.02)$, glucose incremental percentage $(p=0.047)$, area under curve $(p<0.05)$, and glucose peak time ( $p$ $=0.022$ ). We have not found difference on any variable in insulin curves or on glucose incremental velocity. Regarding insulin secretion indices there were no statistical significance in insulinogenic or delta indices, but they became significant after being corrected by insulin resistance $(p=0.008)$. When we evaluated insulin resistance alone, by using HOMA and QUICKI indices and the fasting glucose to insulin index, we have found statistical significance $(p=0.005 ; p=0.005 ; p=0.053)$. Conclusion: Although studying a small sample, we could suggest that individuals with impaired fasting glucose and impaired glucose tolerance are in different stages of diabetes natural history disease. We found out that the best indices of insulin resistance are both HOMA and QUICKI. We also suggest that pancreatic secretion indices should be corrected by the insulin resistance, which could best reflect type 2 diabetes natural history. (Arq Bras Endocrinol Metab 2007;51/9:1498-1505)

Keywords: Insulin resistance; Insulin secretion; OGTT; Impaired glucose tolerance
Recebido em 28/03/06

Revisado em 19/12/06 Aceito em 02/08/07 
A ÍNDROME DE RESISTÊNCIA INSULÍNICA caracterizase pela associação entre obesidade, hipertensão arterial sistêmica (HAS), dislipidemia, doença aterosclerótica, alteração no metabolismo de carboidratos e hiperinsulinemia (1). Seu conceito foi descrito há mais de 80 anos, mas nas últimas duas décadas houve aumento exponencial de sua incidência em todo o mundo, estando associada à epidemia global de obesidade e diabetes mellitus tipo 2 (DM2). Esta síndrome também é conhecida como síndrome metabólica, síndrome X ou "quarteto da morte" (2). A prevalência da resistência insulínica varia de 8 a 43\%, dependendo da população estudada, porém aumenta universalmente com a idade.

A fisiopatologia da resistência insulínica é explicada por uma redução da ação da insulina nos tecidos periféricos, resultando em aumento compensatório da secreção de insulina. $\mathrm{O}$ desequilíbrio deste mecanismo resulta na progressiva diminuição da tolerância à glicose, que pode ter como resultado final o surgimento de DM2 (3). Este dado indica que provavelmente as alterações fisiopatológicas da doença estão presentes vários anos antes de sua expressão clínica.

Indivíduos com resistência insulínica têm risco aumentado de desenvolver outros eventos clínicos além do DM2, como a síndrome dos ovários policísticos, esteatose hepática e doença cardiovascular (2).

As doenças cardiovasculares são a principal causa de mortalidade nos países ocidentais, e o conceito de síndrome metabólica ou síndrome de resistência insulínica é útil na prática clínica para o tratamento e prevenção destas doenças. Fatores genéticos e ambientais estão envolvidos no desenvolvimento da síndrome (4).

O diagnóstico precoce da resistência insulínica é de grande importância, existindo vários métodos para detectá-la. A maioria dos indivíduos com resistência insulínica apresenta, clinicamente, síndrome metabólica. A definição clínica de síndrome metabólica varia entre os continentes, mas todas as classificações têm em comum a presença de obesidade, HAS, intolerância à glicose e dislipidemia (2).

Em relação ao diagnóstico laboratorial, o clamp euglicêmico hiperinsulinêmico é o padrão de referência, mas torna-se inapropriado na prática clínica devido à sua invasividade e complexidade (5). Vários outros parâmetros vêm sendo utilizados, tendo boa correlação com o clamp, como o teste oral de tolerância à glicose (TOTG), índices como o QUICKI (quantitative insulin sensitivity check index), índice HOMA (homeostasis model assessment) e a relação glicose/insulina (6-9).
O diagnóstico precoce da resistência insulínica permite-nos, portanto, tratar precocemente e prevenir as doenças cardiovasculares, principais causas mundiais de mortalidade.

O objetivo principal deste estudo é determinar a associação entre os parâmetros clínicos, demográficos e os índices de secreção e resistência insulínica durante o TOTG sob estímulo com dextrosol 82,5 g em uma população de indivíduos aparentemente saudáveis, sem o conhecimento prévio do seu nível de tolerância à glicose. Após a realização do TOTG, poderemos classificar os indivíduos de acordo com seu metabolismo glicídico como normais, intolerantes de jejum, intolerantes à glicose e/ou diabetes mellitus. A seguir, realizaremos a avaliação dos diferentes índices de secreção e resistência insulínica nesta amostra, comparando os diferentes grupos.

\section{PACIENTES E MÉTODOS}

Foram avaliados 105 indivíduos no período de fevereiro a agosto de 2003, com idade de 33,4 \pm 1,4 (19-65) anos, sendo $57,1 \%$ do sexo feminino $(\mathrm{n}=60), 60 \%$ brancos $(\mathrm{n}=$ 63 ) e nível de escolaridade de $12,5 \pm 0,5(13 ; 1-28)$ anos de estudo (tabela 1). Os anos de estudo foram considerados a partir da alfabetização do indivíduo.

Todos os indivíduos foram submetidos a um inquérito clínico-demográfico, onde o índice de massa corporal (IMC) foi calculado dividindo-se o peso $(\mathrm{Kg})$ pela altura ao quadrado $\left(\mathrm{m}^{2}\right)$, a pressão arterial sistêmica (PA) foi aferida após 5 minutos de repouso com esfigmomanômetro de coluna de mercúrio padronizado e calibrado. Para a determinação da relação cintura/quadril (RCQ), foram realizadas aferições com fita métrica a meio caminho entre o rebordo costal e ao nível da cicatriz umbilical (cintura) e ao nível da proeminência do osso ilíaco (quadril), com repetição das aferições após intervalo de cinco minutos. Consideramos obesidade andróide uma relação $\geq$ 0,95 para homens $\mathrm{e} \geq 0,85$ para mulheres.

Os indivíduos foram submetidos ao teste oral de tolerância à glicose (TOTG) com 82,5 gramas de dextrosol após jejum noturno de 12 horas; na véspera, foram orienta-

Tabela 1. Características da população.

\begin{tabular}{lc} 
Idade (anos) & $33,4 \pm 1,4$ \\
Sexo (F/M) & $60 / 45$ \\
Cor (B/NB) & $63 / 42$ \\
\hline IMC (Kg/m²) & $24,9 \pm 0,4$ \\
PAS (mmHg) & $115,9 \pm 1,6$ \\
PAD (mmHg) & $72,8 \pm 0,9$ \\
Escolaridade (anos de estudo) & $12,5 \pm 0,5$ \\
\hline
\end{tabular}

PAS: pressão arterial sistólica, PAD: pressão arterial diastólica, IMC: índice de massa corpórea, F: feminino, M: masculino, B: brancos, NB: não brancos. 
dos a não realizar atividade física vigorosa e a realizar dieta com mais de 150 gramas de carboidratos/dia durante 5 dias. $\mathrm{O}$ acesso venoso periférico foi realizado por punção de veia de membros superiores e mantido com infusão contínua de solução de cloreto de sódio na concentração de $0,9 \%$.

As coletas de sangue para dosagem da glicemia e da insulina foram realizadas nos tempos zero (imediatamente antes da solução de dextrosol), trinta, sessenta e cento e vinte minutos após a ingestão da solução. Durante todo o período da coleta das amostras sanguíneas, os indivíduos estudados foram orientados a permanecer em repouso na posição de decúbito dorsal, em ambiente com temperatura de $22^{\circ}$ Celsius.

As determinações sanguíneas foram analisadas pelos seguintes métodos: glicemias pelo método glicose oxidase; $\mathrm{HbAlc}$ pela técnica de cromatografia líquida de alta precisão no aparelho L-9100 Merck Hitachi (VR: 2,6 a 6,2\%), sendo o coeficiente de variação intra-ensaio para valores baixos $(4,5 \%)$ e altos $(10 \%)<1 \%$; colesterol total, HDL e triglicerídeos através de reações colorimétricas com leitura pelo aparelho Cobas-Mira (Roche); colesterol-LDL foi calculado pela fórmula de Friedwald; ácido úrico através de reação colorimétrica. As insulinemias foram dosadas pelo método de radioimunoensaio (RIA) (DPC-Diagnostic Products Corporation - L.A.). Os valores de referência são de 5-25 $\mu \mathrm{U} / \mathrm{mL}$ e o coeficiente de variação intra-ensaio é de $9 \%$ para valores altos e de 9,8\% para valores baixos.

Foram analisadas as seguintes variáveis nas curvas de glicose e insulina: glicose basal (GB), obtida no tempo zero; valor de pico da glicose (VPg), definido como sendo o maior valor acima do basal observado após ingestão da solução padrão e expresso em $\mathrm{mg} / \mathrm{dL}$; incremento absoluto da gli$\operatorname{cose}(\Delta \mathrm{g})$, definido como sendo a diferença absoluta entre o valor máximo da glicose obtido após estímulo (VPg) e o valor basal $(\mathrm{GB})$, expresso em $\mathrm{mg} / \mathrm{dL}(\Delta \mathrm{g}=\mathrm{VPg}-\mathrm{GB})$; incremento percentual da glicose (PIg), definido como sendo a relação entre o incremento absoluto da glicose $(\Delta \mathrm{g})$ e o valor basal (GB), e expresso em termos percentuais $(\mathrm{PIg}=(\Delta \mathrm{g} / \mathrm{GB}) \times 100)$. Área total abaixo da curva da glicose (ATG), definida como sendo a área abaixo da curva da glicose, até o eixo das abscissas. Foi obtida através do cálculo numérico da integral da curva e expressa em mg/dL.min; velocidade de incremento da glicose (VIG), definida como sendo a relação entre o incremento Absoluto da Glicose $(\Delta \mathrm{g})$ e o tempo, em minutos, onde foi registrado o valor de pico (TPG), e expressa em $\mathrm{mg} / \mathrm{ml} \cdot \mathrm{min}^{-1}$ (VIP $=\Delta \mathrm{p} /$ tempo de pico). Nos pacientes cujas curvas não apresentaram incremento, ou que apresentaram valores após estímulo menores que o basal, o $\Delta \mathrm{g}$, o PIG e a VIG foram considerados como zero.

Para avaliação do grau de secreção e resistência insulínica, utilizamos os seguintes índices: indice HOMA, calculado a partir dos níveis de glicemia basal e insulinemia basal, segundo a fórmula: insulina basal x glicemia basal ( $\mathrm{mmol}) / 22,5$, e expresso em pontos percentuais; indice insulinogênico: (insulina30'-insulina basal)/(glicemia 30'glicemia basal), expresso em $\mathrm{mU} \cdot \mathrm{mmol}^{-1}$; índice delta: (insulina 30'-insulina basal)/glicemia 30', também expresso em mU.mmol-1; indice QUICKI: calculado segundo a seguinte fórmula: $1 / \log$ insulina em $\operatorname{jejum}(\mathrm{mU} / \mathrm{L})+\log$ glicemia em jejum $(\mathrm{mg} / \mathrm{dL})$; relação glicose/insulina em jejum, expressa em $\mathrm{mg} / 10^{-4} \mathrm{UI}$; relação entre os indices insulinogênico e HOMA: foi utilizado para comparar a relação entre secreção e resistência insulínica, sendo expresso $\mathrm{em} 1 / \mathrm{mmol}^{2}$.

Os indivíduos foram inicialmente divididos em 2 grupos de acordo com o IMC: normais, quando IMC era $<25$ ou sobrepeso/obesos se este fosse $\geq 25 \mathrm{Kg} / \mathrm{m}^{2}$. Posteriormente, foram subdivididos de acordo com o grau de tolerância à glicose em: normais, quando glicemia em jejum $(\mathrm{GJ})<100$ e glicemia 120 minutos (G2h) após administração de dextrosol < 140, intolerância de jejum quando GJ $\geq 100$ e $\leq 126$ e intolerância oral à glicose e/ou diabetes mellitus se $\mathrm{G} 2 \mathrm{~h} \geq 140 \mathrm{mg} / \mathrm{dL}$.

Os dados foram analisados no programa EPI-INFO versão 2000 para DOS, sendo complementado pelo SPSS (Statistical Package for the Social Sciences) para Windows versão 10.0, 2001. Todas as variáveis foram testadas quanto a sua normalidade pelo teste de Kolmogorov-Smirnov (K-S).

Os resultados foram apresentados como média \pm desvio-padrão para as variáveis com distribuição normal e como mediana (mínimo e máximo) para as variáveis nãoGaussianas.

Os seguintes testes estatísticos foram utilizados: Teste $t$ para comparação de duas médias, quando a variável em análise apresentava distribuição normal, e teste não paramétrico de Mann-Whitney $(\mathrm{Z})$, quando a distribuição era anormal. O intervalo de confiança adotado foi de $95 \%$.

Para comparação das médias dos pontos de três amostras foram utilizadas análises de variância $(\mathrm{F})$, quando a variável tinha distribuição normal, e o teste de Kruskal-Wallis $(\mathrm{H})$, quando a distribuição era anormal, e, para avaliarmos entre quais das três amostras ocorria a diferença, foi realizada a comparação dos grupos dois a dois, através de teste paramétrico e não paramétrico para variáveis com e sem distribuição normal, respectivamente e utilizando a correção de Bonferroni com nível de significância $\mathrm{p}<0,012$.

Para a comparação de distribuição de freqüência de variáveis categóricas de amostras categóricas de variáveis independentes utilizamos o teste exato de Fisher; no caso de avaliação de freqüência de variáveis entre três amostras, foi utilizado o teste do Chi-quadrado.

As correlações de Pearson (r) e Spearman (rho) foram utilizadas para avaliar o grau de correlação entre as variáveis contínuas de distribuição normal e anormal, respectivamente.

O teste de regressão múltipla em stepwise foi utilizado para análise de correlação entre três ou mais variáveis contínuas selecionadas quando apresentavam $\mathrm{p}<0,10$ na correlação de Pearson ou Spearman, adotando-se um intervalo de confiança de 95\%. As variáveis sem distribuição normal entraram no modelo após transformação logarítmica. 


\section{RESULTADOS}

Da amostra geral, estratificamos grupos de indivíduos, de acordo com o IMC e o grau de tolerância à glicose, sendo observados $55(52,4 \%)$ indivíduos normais (grupo 0), 29 (27,6\%) indivíduos com metabolismo normal com sobrepeso e/ou obesos (grupo 1), $9(8,6 \%)$ intolerantes de jejum (grupo 2/IFG) e 12 (11,4\%) com intolerância oral à glicose (IGT) e/ou DM 2 (grupo 3), independentemente do nível do IMC.

Comparando os subgrupos de indivíduos, encontramos diferença na idade, respectivamente, para os grupos $0,1,2$ e $3(26,7 \pm 9,3$ vs. $34,9 \pm 15,2$ vs. 53,1 $\pm 9,1$ vs. $45,5 \pm 14,8$ anos; $\mathrm{p}<0,05)$.

A medida da cintura foi diferente entre os 4 subgrupos: $73,9 \pm 8,6$ vs. $88,7 \pm 11,1$ vs. $92,6 \pm 10,4$ vs. $90,3 \pm 15,2 \mathrm{~cm}(\mathrm{p}<0,05)$, encontrando-se tendência para RCQ: $0,80 \pm 0,06$ vs. $0,88 \pm 0,15$ vs. $0,87 \pm 0,12$ vs. $0,85 \pm 0,09(\mathrm{p}=0,052)$. O IMC foi diferente entre os 4 subgrupos $(\mathrm{p}<0,05)$, porém, após análise intragrupos, manteve-se significativo apenas entre o grupo normal e qualquer um dos demais subgrupos, não se encontrando diferença entre indivíduos obesos, IFG ou IGT/DM2.

Encontramos diferença significativa tanto para a GJ quanto para a G2h entre os 4 grupos, respectivamente, GJ: $80,1 \pm 9,9$ vs. $84,9 \pm 10,2$ vs. $106,1 \pm 7,4$ vs. $102,4 \pm 13,8 \mathrm{mg} / \mathrm{dL}(\mathrm{p}<0,05)$; G2h: $94,4 \pm 19,8$ vs. $96,4 \pm 19,3$ vs. $108,7 \pm 13,9$ vs. $167,0 \pm 18,1$ $\mathrm{mg} / \mathrm{dL}(\mathrm{p}<0,05)$. Não houve diferença estatística para as insulinemias em jejum ou em 2 horas $(\mathrm{p}=$ 0,083 e $\mathrm{p}=0,85$, respectivamente).

Em relação às variáveis analisadas na curva de glicose durante o TOTG, encontramos diferença estatisticamente significante para o valor de pico da glicose $(\mathrm{p}<0,05)$, delta de glicose $(\mathrm{p}<0,05)$, percentual de incremento de glicose $(\mathrm{p}=0,047)$, área sob a curva de glicose (figura 1$)(\mathrm{p}<0,05)$ e tempo de pico de glicose $(\mathrm{p}=0,022)$. Não encontramos diferença para a velocidade de incremento de glicose $(\mathrm{p}=0,59)$, assim como para nenhuma variável analisada na curva de insulina. Os dados estão descritos na tabela 2.

Ao avaliarmos os índices de secreção e resistência insulínica (tabela 3), encontramos diferença entre os quatro grupos para o índice HOMA $(\mathrm{p}=0,005)$, índice insulinogênico (NS), índice delta (NS), índice QUICKI $(\mathrm{p}=0,005)$, relação glicose/insulina em jejum (NS) e relação entre os índices insulinogênico e de HOMA ( $\mathrm{p}=0,008)$ (figuras 2, 3 e 4).

$\mathrm{Na}$ análise multivariada em stepwise, para o grupo geral, ao considerarmos a área sob a curva de glicose (ATG) como variável dependente e idade, IMC, anos de estudo, sexo masculino, GJ, G2h, insulina basal (IB) e insulina em 2 horas (I2h) como variáveis independentes, encontramos correlação direta com a G2h $\left(\mathrm{r}=0,80 ; \mathrm{r}^{2}=\right.$ $0,64 ; \mathrm{p}<0,05)$, GJ $\left(\mathrm{r}=0,86 ; \mathrm{r}^{2}=0,74 ; \mathrm{p}<0,05\right)$, correlação inversa com anos de estudo $\left(r=0,87 ; r^{2}=0,76\right.$; $\mathrm{p}<0,05)$ e correlação direta com I2h $\left(\mathrm{r}=0,88 ; \mathrm{r}^{2}=\right.$ $0,77 ; \mathrm{p}<0,05)$. Nos grupos 0 e 1 , de indivíduos com tolerância normal à glicose, considerando ATG como variável dependente e as mesmas variáveis como independentes, encontramos correlação com G2h $(\mathrm{r}=0,72$; $\left.\mathrm{r}^{2}=0,52 ; \mathrm{p}<0,05\right), \mathrm{GJ}\left(\mathrm{r}=0,8 \mathrm{l} ; \mathrm{r}^{2}=0,65 ; \mathrm{p}<0,05\right)$, idade $\left(\mathrm{r}=0,82 ; \mathrm{r}^{2}=0,68 ; \mathrm{p}<0,05\right)$ e I2h $\left(\mathrm{r}=0,84 ; \mathrm{r}^{2}\right.$ $=0,70 ; \mathrm{p}<0,05)$. Ao analisarmos os grupos 2 e 3 , constituídos por indivíduos com disglicemia, considerando ATG como variável dependente e as mesmas variáveis como independentes, encontramos correlação com G2h $\left(\mathrm{r}=0,7 \mathrm{l} ; \mathrm{r}^{2}=0,5 \mathrm{l} ; \mathrm{p}<0,05\right)$, correlação inversa com anos de estudo $\left(r=0,86 ; r^{2}=0,76 ; p<0,05\right)$ e correlação direta com o sexo masculino $\left(\mathrm{r}=0,92 ; \mathrm{r}^{2}=0,84 ; \mathrm{p}<\right.$ $0,05)$. Após excluirmos GJ e G2h das variáveis dependentes, as variáveis mais importantes foram idade e IMC.

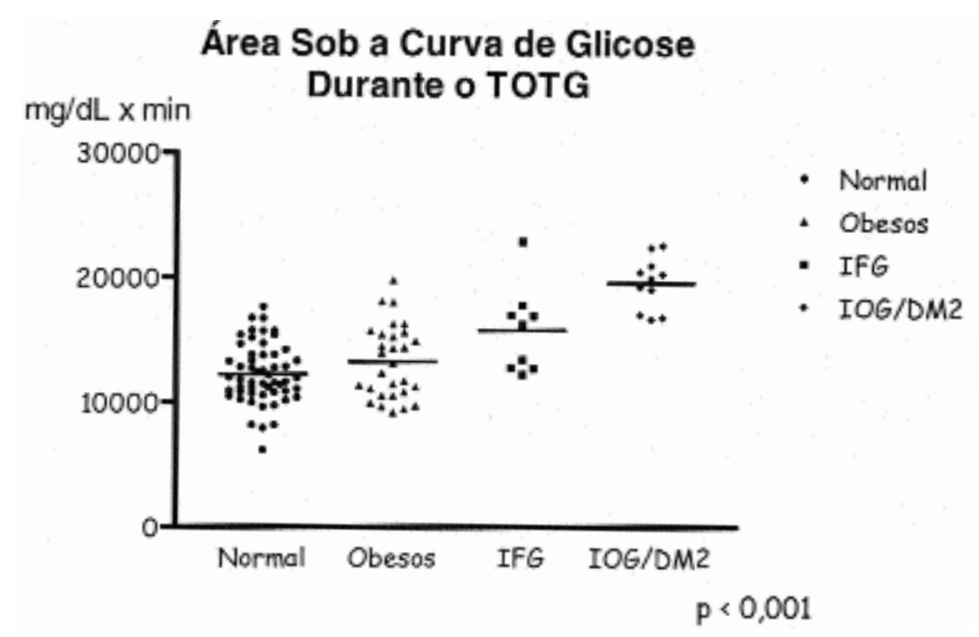

Figura 1. Área sob a curva de glicose durante o TOTG. 
Tabela 2. Variáveis analisadas nas curvas de glicose e insulina durante o TOTG.

\begin{tabular}{|c|c|c|c|c|c|}
\hline Variável/Grupo & 0 & 1 & 2 & 3 & $p$ \\
\hline $\mathrm{GJ}(\mathrm{mg} / \mathrm{dL})$ & $80,1 \pm 9,9^{*}$ & $84,9 \pm 10,2 \triangleleft / \otimes \infty$ & $106,1 \pm 7,4$ & $102,4 \pm 13,8$ & $<0,05$ \\
\hline G2h (mg/dL) & $94,4 \pm 19,8 \#$ & $96,5 \pm 19,3 \# \#$ & $108,7 \pm 13,9$ \#\#\# & $167,0 \pm 18,1$ & $<0,05$ \\
\hline $\operatorname{VPg}(\mathrm{mg} / \mathrm{dL})$ & $123,6 \pm 27,1 \bullet / \bullet$ & $132,97 \pm 34,6 \cdots$ & $162,6 \pm 46,4$ & $190,6 \pm 19,8$ & $<0,05$ \\
\hline$\Delta \mathrm{g}(\mathrm{mg} / \mathrm{dL})$ & $43,67 \pm 27,8^{\circ}$ & $48,0 \pm 30,4^{\circ \circ}$ & $56,4 \pm 41,26$ & $86,4 \pm 22,13$ & $<0,05$ \\
\hline ATG (mg/dL.min) & $12181,39 \pm 2385,28 \ddagger / \neq \neq$ & $13180,35 \pm 2921,40$ 抽 & $15678,33 \pm 3436,49$ & $19479,55 \pm 2091,33$ & $<0,05$ \\
\hline Pig (\%) & $56,66 \pm 39,34 \S$ & $56,21 \pm 33,0 \S \S$ & $51,95 \pm 36,5$ & $85,1 \pm 25,6$ & 0,047 \\
\hline TPG (min) & $41,66 \pm 23,61 \dagger$ & $46,48 \pm 26,13$ & $40,0 \pm 15,0$ & $73,6 \pm 38,8$ & 0,022 \\
\hline $\mathrm{IJ}(\mu \mathrm{U} / \mathrm{mL})$ & $7,9 \pm 3,8$ & $10,3 \pm 5,1$ & $7,8 \pm 2,6$ & $10,1 \pm 4,4$ & 0,083 \\
\hline $\mathrm{I} 2 \mathrm{~h}(\mu \mathrm{U} / \mathrm{mL})$ & $36,74 \pm 30,67$ & $41,73 \pm 36,53$ & $30,83 \pm 19,75$ & $53,23 \pm 65,72$ & 0,847 \\
\hline
\end{tabular}

GJ: glicemia de jejum, G2h: glicemia 120 minutos após administração de 75 gramas de glicose anidra, VPg: valor de pico da glicose, $\Delta$ : delta da glicose, ATG: área abaixo da curva de glicose, Pig: percentual de incremento da glicose, TPG: tempo de pico da glicose, IJ: insulinemia de jejum, I2h: insulinemia $2 \mathrm{~h}$ após administração de glicose anidra.

${ }^{*} p=0,0000$, grupo 0 vs. 1 e grupo 0 vs. $2 ; \diamond p=0,0000$, grupo 1 vs. $2 ; \otimes p=0,0006$, grupo 1 vs. $3 ; \# p=0,0000$, grupo 0 vs. 3; \#\# $\mathrm{p}=0,0000$, grupo 1 vs. 3; \#\#\# $\mathrm{p}=0,0000$, grupo 2 vs. $3 ; \quad \mathrm{p}=0,0101$, grupo 0 vs. $2 ; \cdots p=0,0000$, grupo 0 vs. $3 ; \cdots p=0,0000$, grupo 1 vs. $3 ;{ }^{\circ} p=0,0000$, grupo 0 vs. $3 ;{ }^{\circ} p=0,0006$, grupo 1 vs. $3 ; \neq p=0,0023$, grupo 0 vs. $2 ; \neq \neq p=0,0000$, grupo 0 vs. $3 ; \neq \neq \ddagger$ $\mathrm{p}=0,0000$, grupo 1 vs. $3 ; \S p=0,0101$, grupo 0 vs. 3 ; $\S \S p=0,0088$, grupo 1 vs. 3 ; $\uparrow p=0,0025$, grupo 0 vs. 3 .

Tabela 3. Índices de secreção e resistência insulínica.

\begin{tabular}{|c|c|c|c|c|c|}
\hline Variável/Grupo & 0 & 1 & 2 & 3 & $p$ \\
\hline HOMA (\%) & $1,58 \pm 0,78$ * & $2,19 \pm 1,18$ & $2,06 \pm 0,77$ & $2,62 \pm 1,22$ & 0,005 \\
\hline Índice Insulinogênico (mU.mmol-1) & $190,83 \pm 318,43$ & $385,61 \pm 1199,41$ & $330,05 \pm 692,77$ & $96,08 \pm 144,02$ & 0,21 \\
\hline Índice Delta (mU.mmol-1) & $43,42 \pm 28,42$ & $41,86 \pm 31,05$ & $31,66 \pm 14,69$ & $32,63 \pm 22,21$ & 0,52 \\
\hline QUICKI & $0,366 \pm 0,0031 \S / \S \S$ & $0,347 \pm 0,024$ & $0,347 \pm 0,021$ & $0,339 \pm 0,032$ & 0,005 \\
\hline Glicose/Insulina (mg/10-4 UI) & $12,76 \pm 7,2$ & $9,8 \pm 4,0$ & $15,11 \pm 5,3$ & $12,12 \pm 5,8$ & 0,053 \\
\hline Insulinogênico/HOMA (1/mmol²) & $180,16 \pm 327,71 \dagger$ & $140,56 \pm 263,64+\dagger$ & $149,91 \pm 261,07$ & $41,56 \pm 77,68$ & 0,008 \\
\hline
\end{tabular}

${ }^{*} p=0,0053$, grupo 0 vs. $3 ; \S p=0,0118$, grupo 0 vs. 1 ; $\S \S p=0,0056$, grupo 0 vs. $3 ; \dagger p=0,0010$, grupo 0 vs. 3 ; $\dagger^{\dagger} p=0,0105$, grupo 1 vs. 3.

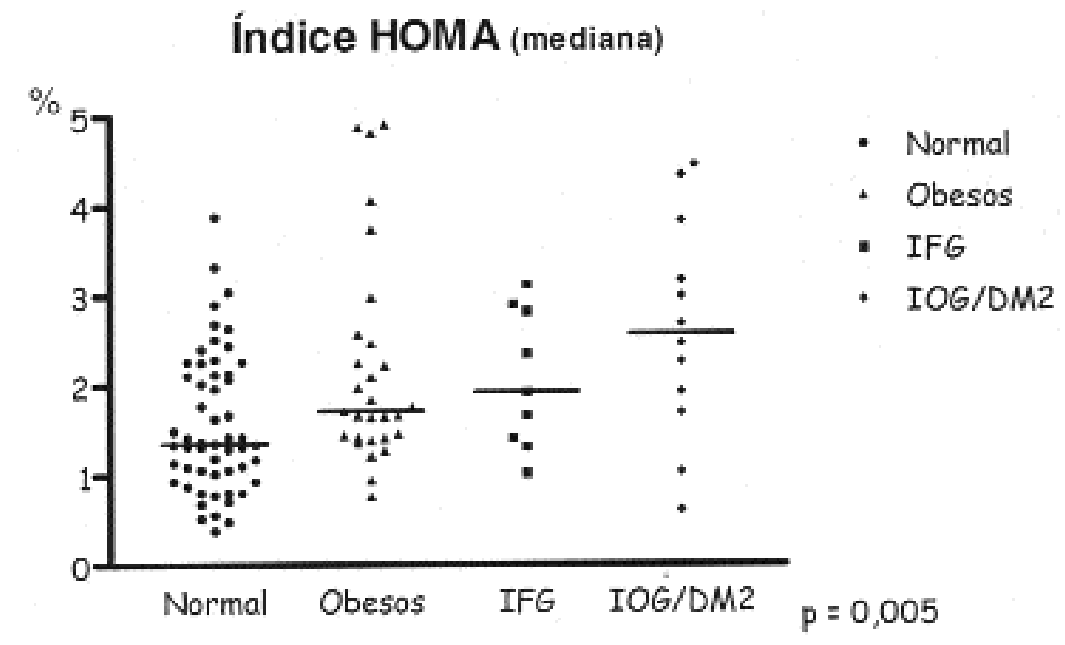

Figura 2. Índice HOMA. 


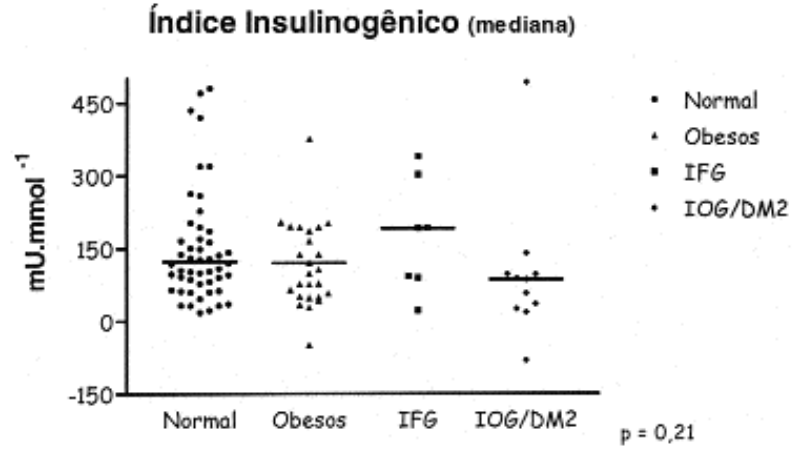

Figura 3. Índice insulinogênico.

\section{DISCUSSÃo}

A obesidade é freqüentemente associada à resistência insulínica, sendo que ambas são fatores de risco para o surgimento de DM2 e doenças cardiovasculares. O DM2 resulta da combinação de resistência insulínica e déficit de secreção de insulina. Durante sua história natural, à medida que surge alteração da glicemia de jejum ou intolerância à glicose, já parece haver disfunção da célula beta, com perda considerável da secreção insulínica.

Enfatizamos que, em nossa população de indivíduos jovens aparentemente saudáveis, encontramos $20 \%$ da amostra com algum grau de intolerância à glicose, seja em jejum $(8,6 \%)$ ou pós-prandial $(11,4 \%)$.

Existem várias formas de avaliação da secreção e resistência insulínica, conforme descrição nos métodos acima. Em nosso estudo, realizamos o TOTG com 75 gramas de glicose anidra, analisando as variáveis obtidas durante as curvas de glicose e insulina, além dos índices também já descritos, que já foram validados pela literatura. Todas as variáveis analisadas na curva de glicose durante o TOTG, com exceção da velocidade de incremento da glicose, foram diferentes entre os grupos, sugerindo que, durante a história natural da intolerância oral à glicose até DM2, ocorra gradativamente redução da secreção insulínica quando corrigido para o grau de resistência insulínica (10).

Em nossa análise multivariada, observamos que, nos indivíduos com metabolismo glicídico ainda normal, mesmo quando obesos, existe correlação da glicemia de jejum e da insulinemia em 2 horas com a área sob a curva de glicose, não havendo diferença para aqueles indivíduos com glicemia de jejum alterada, intolerância à glicose ou diabetes mellitus. Nestes últimos, apenas a glicemia em 2 horas é preditora da área sob a curva de glicose, sugerindo perda da primeira fase de secreção insulínica.
Secreção / Resistência (mediana)

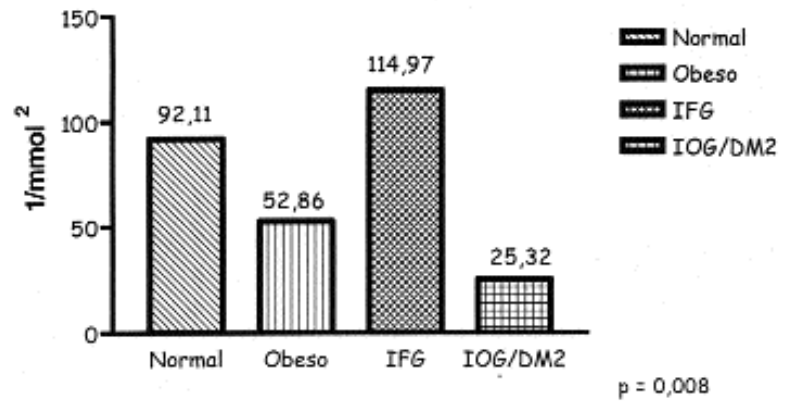

Figura 4. Relação entre os índices insulinogênico e HOMA.

A primeira fase da secreção insulínica, quando reduzida, leva ao aumento da glicemia pós-prandial ou em 2 horas.

A hiperglicemia de jejum é um evento tardio na história natural do DM2 e a disfunção da célula beta pancreática é progressiva, o que sugere que a intolerância à glicose ocorre anteriormente à hiperglicemia de jejum (11). A cada aumento de 50\% na resistência insulínica muscular, o pâncreas aumenta a secreção de insulina em duas vezes. Entretanto, nos indivíduos com intolerância oral à glicose e DM2 ocorre aumento de apenas $15 \%$ da secreção insulínica em resposta ao aumento da resistência insulínica, o que resulta em hiperglicemia (12).

Apenas dois indivíduos em nossa amostra preencheram critérios diagnósticos para diabetes mellitus $(1,9 \%)$, sendo um deles pela glicemia de jejum, e outro após sobrecarga glicídica, ambos com idade acima de 40 anos e IMC $\geq 27 \mathrm{Kg} / \mathrm{m}^{2}$. Os dois indivíduos apresentaram índice insulinogênico muito inferior à média $(57,5$ e 88,8 mU.mmol-1), confirmando que possivelmente a redução da primeira fase de secreção insulínica seja necessária para a ocorrência de DM2, em concomitância à resistência insulínica.

Não encontramos diferença para as variáveis analisadas durante a curva de insulina, o que é concordante com a literatura no que diz respeito a indivíduos com alteração do metabolismo de carboidratos (13). O aumento da insulinemia de jejum parece ser um marcador de resistência insulínica em indivíduos normais, havendo aumento relativo do seu nível circulante $(14,15)$. Entretanto, isto é limitado pela capacidade de secreção pancreática, que, por sua vez, é modificada pela glicemia e pelo clearance de insulina $(14,15)$. Por este motivo, a insulinemia de jejum é um marcador de menor importância em indivíduos com intolerância à glicose ou diabetes mellitus, insulinomas ou patologias que alterem o clearance de insulina (16). 
Quanto aos índices de resistência insulínica, encontramos diferença estatisticamente significativa para o HOMA e o QUICKI, que possuem boa correlação com o clamp euglicêmico hiperinsulinêmico entre os grupos estudados $(7,8)$. Determinações do índice QUICKI com valores $<0,357$ apresentam correlação com a síndrome metabólica, o está de acordo com nossos achados para os grupos de obesos e indivíduos com alteração do metabolismo glicídico (18).

Existem divergências na literatura quanto à utilização do índice HOMA em indivíduos com graus alterados de tolerância à glicose e diabetes mellitus $(19,20)$. Entretanto, a maioria dos autores é a favor da utilização deste índice, uma vez que permite a discriminação entre os indivíduos com tolerância à glicose normal, alterada e DM2 (6). A partir do momento em que existe hiperglicemia em jejum, o índice HOMA pode superestimar a RI, mas nem por isso deve deixar de ser utilizado (21). Modelos informatizados mais recentes do índice HOMA permitem seu uso para variações de insulinemia de $0,1-317 \mu \mathrm{U} / \mathrm{mL}$ e de glicemia de $18-450 \mathrm{mg} / \mathrm{dL}(22)$. O índice vem sendo utilizado para avaliar modificações longitudinais na função da célula beta e na resistência insulínica para avaliar a história natural do DM e em indivíduos com DM2 para avaliar os efeitos do tratamento (23). O índice HOMA é preferencialmente utilizado em grandes estudos epidemiológicos, tendo sido avaliado em mais de 150 estudos com indivíduos de diversas origens étnicas e diferentes graus de tolerância à glicose, constituindo-se importante meio de avaliação da resistência insulínica em qualquer população (22). Entretanto, até o presente momento, não se determinou um ponto de corte que defina a resistência insulínica.

Recentemente, alguns trabalhos mostraram que seria mais importante ajustar os índices de secreção pelo grau de resistência insulínica, corrigindo desta forma todas as diferenças pré-existentes entre diferentes grupos étnicos (24). Em nossa amostra, optamos por ajustar a secreção em relação à resistência insulínica ao corrigirmos o índice HOMA pelo índice insulinogênico, encontrando diferença entre os grupos estudados (25). Apesar de as determinações de insulinemias não serem estatisticamente significativas, após esta correção encontramos diferença entre os grupos, demonstrando que o déficit de insulina nos indivíduos com distúrbio no metabolismo glicídico é relativo e não absoluto.

A primeira alteração encontrada na fisiopatologia do diabetes tipo 2 é a resistência insulínica, sendo mais tardio o achado de alteração da secreção pancreática. Estudos epidemiológicos mostram que é importante considerar o grau de resistência insulínica na avaliação da secreção insulínica, e que ambos são fatores de risco independentes para o desenvolvimento de DM2 (26).

Apesar de o tamanho de nossa amostra nos diferentes grupos de intolerância à glicose ser uma limitação do nosso estudo, podemos inferir que indivíduos com intolerância de jejum à glicose e indivíduos com intolerância pós-prandial possivelmente estão em momentos diferentes da história natural da doença.

Concluímos pelos nossos dados que os melhores índices para a avaliação de resistência insulínica são o HOMA e o QUICKI, e que os índices de avaliação da secreção pancreática devem ser corrigidos para o grau de resistência insulínica, de modo a refletir melhor a história natural do diabetes mellitus.

\section{REFERÊNCIAS}

1. Reaven GM. Banting Lecture 1988: role of insulin resistance in human disease. Diabetes Care 1988;37:1595-607.

2. Ecket RH, Grundy SM, Zimmet PZ. The metabolic syndrome. Lancet 2005;365:1415-28.

3. Defronzo RA, Ferrannini E. Insulin resistance: a multifaceted syndrome responsible for NIDDM, obesity, hypertension, dyslipidemia and atherosclerotic cardiovascular disease. Diabetes Care 1991;14:173-94.

4. Grundy SM, Brewer HB, Cleeman JI, Smith SC, Lenfant C. Definition of metabolic syndrome. Circulation 2004;109:433-8.

5. Defronzo RA, Tobin JD, Andres R. Glucose clamp technique: a method for quantifying insulin secretion and resistance. Am J Physiol 1979;237:E214-33.

6. Corcoy R, Leiva A, Murugo M, Espinosa RJ, Albareda M. Assessment of insulin sensitivity and beta cell function from measurements in the fasting state and during an oral glucose tolerance test. Diabetologia 2000;43:1507-11.

7. Katz A, Sridhar SN, Kieren M, Baron AD, Follman DA, Sullivan G, et al. Quantitative insulin sensitive check index: a simple, accurate method for assessing insulin sensitivity in humans. J Clin Endocrinol Metab 2000;85(7):2402-10.

8. Bonora E, Targher G, Alberiche M, Bonadonna RC, Saggiani $\mathrm{F}$, Zenere MB, et al. Homeostasis model assessment closely mirrors glucose clamp technique in the assessment of insulin sensitivity. Diabetes Care 2000;23(1):57-63.

9. Dunaif $A$, Legro R, Finegoog D. A fasting glucose to insulin ratio is a useful measure of insulin sensitivity in women with polycystic ovary syndrome. J Clin Endocrinol Metab 1998;83(8):2694-8.

10. Del Prato S. Loss of early insulin secretion leads to postprandial hyperglycaemia. Diabetologia 2003;46(suppl. 1):M2-8.

11. Kahn SE. Clinical review 135 . The importance of $\beta$-cell failure in the development and progression of type 2 diabetes. $\mathbf{J}$ Clin Endocrinol Metab 2001;86(9):4047-58.

12. Ferranini E, Gastadelli A, Miyazaki Y, Matsuda M, Mari A, Defronzo $R$. $\beta$-cell function in subjects spanning the range from normal glucose tolerance to overt diabetes: a new analysis. J Clin Endocrinol Metab 2005;90(1):493-500.

13. Mooy JM, Grootenhuis PA, Vries H, Bouter LM, Kostense PJ, Heine RJ. Determinants of specific serum insulin concentrations in a general Caucasian population aged 50 to 74 years (The Hoorn Study). Diabet Med 1998;15:45-52.

14. Nguyen L, Vague P. Rationale and methods for the estimation of insulin secretion in a given patient. Diabetes 2001; 51(suppl. 1):S240-4. 
15. Mcauley KA, Williams SM, Mann JI, Walker RJ, Barned NJ, Temple LA, et al. Diagnosing insulin resistance in general population. Diabetes Care 2001;24(3):460-4.

16. Savage $P$, Bergman R, Selby JV, Saad MF, Haffner S, Zaccaro $D$, et al.; for the Insulin Resistance Atherosclerosis Study Investigations (IRAS). Insulin sensitivity, insulinemia, and coronary artery disease. Diabetes Care 2004;27(3):781-7.

17. Phillips DIW, Clack PM, Hales CN, Osmond C. Understanding oral glucose tolerance: comparison of glucose or insulin measurements during the oral glucose tolerance test with specific measurements of insulin resistance and insulin secretion. Diabet Med 1994:11:286-92.

18. Cizek L, Horakova D, Malincikova J, Janout V, Hrebicek J. Detection of insulin resistance by simple quantitative insulin sensitivity check index QUICKI for epidemiological assessment and prevention. J Clin Endocrinol Metab 2002;87(1):144-7.

19. Defronzo RA, Matsuda M. Insulin sensitivity indices obtained from oral glucose tolerance testing. Comparison with the euglycemic insulin clamp. Diabetes Care 1999;22(9):146270.

20. Haffner SM, Saad MF, Karter AJ, Hanley AJ, Agostino R, Festa A. Differences in insulin resistance in nondiabetic subjects with isolated impaired glucose tolerance or isolated impaired fasting glucose. Diabetes 2004;53:1549-55.

21. Tripathy $D$, Carlson $M$, Almgren $P$, Isomaa $B$, Taskinen MR, Tuomi T, et al. Insulin secretion and insulin sensitivity in relation to glucose tolerance. Lessons from the Botnia study. Diabetes 2000;49:975-80.
22. Matthews DR, Levy JC, Wallace TM. Use and abuse of HOMA modeling. Diabetes Care 2004;27(6):1487-95.

23. UKPDS 5. Characteristics of newly presenting type 2 diabetic patients: estimated insulin sensitivity and islet $\beta$-cell function - Multi-centre study. Diabet Med 1988;5(5):444-8.

24. Kahn SE, Fujimoto WY, Hull RL, Cnop M, Jensen CC; and the ADA Gennid Study Group. $\beta$-cell function is a major contributor to oral glucose tolerance in high-risk relatives of four ethnic groups in the U.S. Diabetes 2002;55:2170-8.

25. Kahn SE. The relative contributions of insulin resistance and beta-cell dysfunction to the pathophysiology of type 2 diabetes - Review. Diabetologia 2003;46:3-19.

26. Haffner SM, Miettinen H, Stern MP. The homeostasis model in the San Antonio heart study. Diabetes Care 1997; 20(7):1087-92.

Endereço para correspondência:

Fernanda H. Corrêa

Rua Marechal Ramón Castilla 265/407

22220-090 Rio de Janeiro, RJ 